\title{
A randomized, double-blind, placebo-controlled study of milk oral immunotherapy for cow's milk allergy
}

\author{
Justin M. Skripak, MDa, Scott D. Nash, MD ${ }^{b}$, Hannah Rowley, RD ${ }^{a}$, Nga H. Brereton, RD ${ }^{a}$, \\ Susan Oh, RD ${ }^{\mathrm{a}}$, Robert G. Hamilton, PhD ${ }^{\mathrm{a}}$, Elizabeth C. Matsui, $\mathbf{M D}^{\mathrm{a}}$, A. Wesley Burks, \\ MD $^{\mathrm{b}}$, and Robert A. Wood, MD \\ aDepartment of Pediatrics, Division of Allergy and Immunology, the Johns Hopkins University \\ School of Medicine, Baltimore \\ ${ }^{b}$ Department of Pediatrics, Division of Allergy and Immunology, Duke University Medical Center, \\ Durham
}

\begin{abstract}
Background-Orally administered, food-specific immunotherapy appears effective in desensitizing and potentially permanently tolerizing allergic individuals.

Objective-We sought to determine whether milk oral immunotherapy (OIT) is safe and efficacious in desensitizing children with cow's milk allergy.

Methods-Twenty children were randomized to milk or placebo OIT (2:1 ratio). Dosing included 3 phases: the build-up day (initial dose, $0.4 \mathrm{mg}$ of milk protein; final dose, $50 \mathrm{mg}$ ), daily doses with 8 weekly in-office dose increases to a maximum of $500 \mathrm{mg}$, and continued daily maintenance doses for 3 to 4 months. Double-blind, placebo-controlled food challenges; end-point titration skin prick tests; and milk protein serologic studies were performed before and after OIT.
\end{abstract}

Results-Nineteen patients, 6 to 17 years of age, completed treatment: 12 in the active group and 7 in the placebo group. One dropped out because of persistent eczema during dose escalation. Baseline median milk IgE levels in the active $(n=13)$ versus placebo $(n=7)$ groups were 34.8 $\mathrm{kUa} / \mathrm{L}$ (range, $4.86-314 \mathrm{kUa} / \mathrm{L}$ ) versus $14.6 \mathrm{kUa} / \mathrm{L}$ (range, $0.93-133.4 \mathrm{kUa} / \mathrm{L}$ ). The median milk threshold dose in both groups was $40 \mathrm{mg}$ at the baseline challenge. After OIT, the median cumulative dose inducing a reaction in the active treatment group was $5140 \mathrm{mg}$ (range 2540-8140 $\mathrm{mg})$, whereas all patients in the placebo group reacted at $40 \mathrm{mg}(P=.0003)$. Among 2437 active OIT doses versus 1193 placebo doses, there were 1107 (45.4\%) versus $134(11.2 \%)$ total reactions, with local symptoms being most common. Milk-specific IgE levels did not change significantly in either group. Milk IgG levels increased significantly in the active treatment group, with a predominant milk IgG4 level increase.

Conclusions-Milk OIT appears to be efficacious in the treatment of cow's milk allergy. The side-effect profile appears acceptable but requires further study.

\section{Keywords}

Cow's milk; food allergy; IgE; prognosis; desensitization; tolerance; oral immunotherapy

(C) 2008 American Academy of Allergy, Asthma \& Immunology

Reprint requests: Robert A. Wood, MD, CMSC 1102, The Johns Hopkins Hospital, 600 North Wolfe St, Baltimore, MD 21287. rwood@jhmi.edu.

Disclosure of potential conflict of interest: The rest of the authors have declared that they have no conflict of interest. 
The current standard of care for the management of IgE-mediated food allergy involves the identification of causative foods and avoidance of these allergens while always having selfinjectable epinephrine available. ${ }^{1}$ There are no approved therapies for food allergy, and unfortunately, despite the best efforts of patients to avoid problem foods, accidental exposures are very common. ${ }^{2-4}$ Symptoms associated with these exposures vary, ranging from mild local reactions to life-threatening anaphylaxis. ${ }^{5-8}$

In recent years, orally or sublingually administered allergen-specific immunotherapy has gained increasing attention. Several studies have demonstrated that a substantial number of allergic patients can tolerate gradually increasing amounts of known allergens, including milk, egg, peanut, and hazelnut. ${ }^{9-17}$ The results of these treatments range from protection against small accidental exposures to the ability to tolerate full servings of the allergen. However, the majority of these studies have been small, with limited characterization of patient populations and dosing protocols. Only one, using sublingual immunotherapy for hazelnut allergy, has been undertaken with a randomized, placebo-controlled approach. ${ }^{9}$ The aim of this study was to further investigate the safety and efficacy of oral immunotherapy (OIT) for cow's milk allergy (CMA) in a randomized, double-blind, placebo-controlled study of orally administered milk protein in children with milk allergy.

\section{METHODS}

\section{Study design}

Children between the ages of 6 and 21 years with a known history of IgE-mediated milk allergy were recruited from the pediatric allergy clinics at the Johns Hopkins University Hospital, Baltimore, Maryland, and Duke University Medical Center, Durham, NC. Eligibility criteria were a positive skin prick test (SPT) response to milk extract (wheal $\geq$ histamine control) or milk IgE level of greater than $0.35 \mathrm{kU} / \mathrm{L}$ and a positive milk challenge result at baseline defined as reacting with clear signs, symptoms, or both to a cumulative dose of $2.5 \mathrm{~g}$ or less of milk protein. Patients were excluded if they had a history of anaphylaxis requiring hospitalization, history of intubation related to asthma, or a current diagnosis of severe persistent asthma.

At the baseline visit, a history and physical examination, pulmonary function testing, and end-point titration skin prick testing were performed. Serum was collected for total and milk-specific IgE and IgG antibody measurements (Fig 1). Participants then returned on 2 separate days for a baseline double-blind, placebo-controlled food challenge (DBPCFC) to milk. Participants meeting all eligibility criteria were then randomized in a 2:1 ratio to receive active or placebo OIT.

All initial dosing was conducted in the clinical research facility at both institutions. Milk for active OIT dosing was in the form of dry nonfat powdered milk. All doses were prepared by the clinical research-registered dieticians. The immunotherapy dose schedule is outlined in Table I. On the first day of treatment, a dose escalation was initiated with $0.4 \mathrm{mg}$ of milk protein. Approximately doubling doses were then given every 30 minutes to a maximum of $50 \mathrm{mg}$ (cumulative dose, $98.7 \mathrm{mg}$ ). Participants had to tolerate a minimum dose of $12 \mathrm{mg}$ (cumulative dose, $23.7 \mathrm{mg}$ ) to proceed with home dosing.

Home dosing was then initiated at the highest dose tolerated on the dose escalation day. Premeasured doses were provided to all participants, who were instructed to consume 1 dose per day. All were required to have diphenhydramine and self-injectable epinephrine available at all times. Home diary forms were provided to record the dose date, time taken, symptoms occurring after the dose or any other time, and medications taken each day. After 7 to 14 days on a given dose, participants returned to the research unit to receive a dose 
increase, as described in Table I. Participants who experienced more than a mild reaction with a home dose were seen in the research unit for their next dose, which was typically decreased to the previously tolerated dose. Investigators were available 24 hours a day by telephone for questions or emergencies.

Once a dose of $500 \mathrm{mg}$ (equivalent to $15 \mathrm{~mL}$ of milk) was achieved, participants continued on that dose daily for 13 weeks, after which they underwent their next DBPCFC. Subjects and investigators were unblinded at that time, and those who had been receiving placebo were offered the opportunity to receive open-label active treatment. Subjects receiving active treatment who tolerated less than $2540 \mathrm{mg}$ in this DBPCFC were returned to strict milk avoidance, and the others were given detailed instructions for the continuation of measured amounts of milk protein in their diet based on the challenge outcome.

\section{Study procedures}

End-point titration skin prick testing-End-point titration skin prick testing was conducted with a commercial milk extract (Greer Laboratories, Lenoir, NC) diluted with albumin saline with phenol to concentrations of 1:1, 1:5, 1:10, 1:50, 1:100, 1:1000, $1: 10,000$, and 1:100,000. Testing was performed with a bifurcated needle. Wheals were marked with a ballpoint pen and transferred to records with clear adhesive tape. The most dilute dilution with a wheal at least as large as that elicited by the histamine control was considered the threshold.

Serology-Blood samples were collected before immunotherapy, when the maintenance dose was reached, and after completion of immunotherapy. Serum was analyzed to determine the concentrations (in $\mathrm{kIU} / \mathrm{L}$ ) of total $\operatorname{IgE}$ and the levels (in $\mathrm{kUa} / \mathrm{L}$ ) of $\operatorname{IgE}, \operatorname{IgG}$, and IgG4 antibodies specific for cow's milk (CM) a-lactalbumin, $\beta$-lactoglobulin, and casein by using the Phadia CAP-System FEIA (Phadia Diagnostics, Uppsala, Sweden). CM $\mathrm{IgG}$ and $\mathrm{IgG} 4$ levels could not be directly measured because of the presence of interfering $\operatorname{IgG}$ antibodies specific for bovine albumin in most sera. Thus the sum of the $\mathrm{a}$ lactalbumin-, $\beta$-lactoglobulin-, and casein-specific IgG and IgG4 antibody levels was used as a surrogate measure of $\mathrm{IgG}$ and IgG4 anti-CM antibody level. The lower limit of assay detection was $0.35 \mathrm{kU} / \mathrm{L}$ for the specific IgE assay, $2 \mu \mathrm{g} / \mathrm{mL}$ for the specific $\mathrm{IgG}$ assays, and $0.1 \mu \mathrm{g} / \mathrm{mL}$ for the specific IgG4 assays.

DBPCFC—In the baseline challenge milk doses were 40, 100, 400, 800, and $1200 \mathrm{mg}$, for a cumulative dose of $2.5 \mathrm{~g}$. The available CM-free vehicles were applesauce or soymilk or rice milk flavored with chocolate or strawberry syrup. Either milk powder or placebo powder (Prophree; Abbott Nutrition, Columbus, Ohio) was mixed with the vehicle and administered at the specified doses. Challenges were stopped after clear symptoms of an allergic reaction developed. Emergency medications, including diphenhydramine, epinephrine, albuterol, and prednisone were available and administered at the investigator's discretion.

Postimmunotherapy food challenges followed the same protocol, with the addition of 3 more doses of 1600, 2000, and $2000 \mathrm{mg}$, with a cumulative milk protein dose of $8 \mathrm{~g}$.

\section{Statistical analysis}

Outcomes were evaluated comparing the active and placebo groups. Results of outcomes for placebo-treated participants who subsequently received active therapy are shown separately in selected tables and figures but were not used in the analysis comparing groups. 
All analyses were performed with StataSE 8.0 (StataCorp, College Station, Tex).

Categorical data were expressed as proportions and continuous data as medians with ranges or means with SDs. Proportions in groups were compared by using the Fisher exact test. The Wilcoxon test was used to compare paired data for end-point titration SPT results, total IgE levels, CM IgE levels, and all other serologic measurements (in kilounits per liter), comparing the respective values at baseline with those after treatment. Either the MannWhitney test or the $t$ test was used to compare continuous variables between the 2 groups. A 2-tailed $P$ value of less than .05 was considered statistically significant. Participants who had negative SPT responses at all milk extract concentrations were assigned a threshold concentration value of .1 for the purposes of analysis.

\section{RESULTS}

\section{Study population}

Twenty patients were enrolled in the study (Table II). Seventeen were enrolled at Johns Hopkins Hospital and 3 at Duke University Medical Center. Just over half were male, and the median age was 9 years among the active-treated and 11 years among the placebotreated patients. Most subjects had a past or current history of eczema, asthma, or both, and three fourths had a history of at least 1 other food allergy, with a median of 3 other food allergies. The baseline median milk IgE levels in the active and placebo groups were 34.8 $\mathrm{kUa} / \mathrm{L}$ (range, $4.86-314 \mathrm{kUa} / \mathrm{L}$ ) and $14.6 \mathrm{kUa} / \mathrm{L}$ (range, $0.93-133.4 \mathrm{kUa} / \mathrm{L}$ ), respectively. There were no significant differences between the active and placebo groups.

\section{Milk dose threshold}

The median threshold dose of CM resulting in a reaction was $40 \mathrm{mg}$ in both groups before OIT (Table III and Fig 2). After OIT, the median change in milk dose threshold was 5100 $\mathrm{mg}$ in the active group $(P=.002)$, with no change in the median in the placebo group $(P=$. 16). Among the active treatment group, the range for the threshold amount of dairy eliciting a clear reaction was 2540 to $8140 \mathrm{mg}$. All placebo-treated participants reacted at $40 \mathrm{mg}$ ( $P$ $=.0003)$. Of the 4 participants in the active group to consume the entire $8140 \mathrm{mg}, 2$ experienced no reaction. Because the true threshold was unknown based on this challenge, these 2 are represented in Table III as a threshold of greater than $8140 \mathrm{mg}$. The other 2 experienced mild and untreated symptoms at that maximum dose.

\section{End-point titration SPT threshold}

The median end-point titration SPT threshold concentrations in the active group were 1:50 before treatment and 1:3 after treatment $(P=.03$, Table III and Fig 3$)$. In the placebo group thresholds were 1:100 before treatment and 1:50 after treatment $(P=.03)$. No statistically significant difference was observed in the change from baseline between groups $(P=.12)$.

\section{Serologic studies}

CM-specific IgE antibody results are shown in Table III and Fig 4. Although there was no significant change within any group, there were several individuals in the active group who exhibited marked increases. Some returned to baseline by the post-OIT point, whereas others continued to increase. Although there was no significant change in CM IgG4 levels in the placebo group, there was a median increase from baseline of 767\% in the active group ( $P$ $=.002$, Table III and Fig 5). CM-specific IgG antibody levels increased in parallel with the IgG4 antibody levels. This change from baseline in the active group was statistically significant $(P=.002$, data not shown). These increases were not wholly explained by IgG4 increases, implying that other IgG subclasses were responsible for some of the increase in 
the total CM-specific IgG response. Total serum IgE levels did not change, on average, but some individuals exhibited increases, which is similar to CM-specific IgE patterns.

\section{Safety data}

The results of dose-related safety measurements during double-blind treatment are shown in Table IV. The median number of doses per participant was 177 in the active group and 171 in the placebo group, with a total number of 2437 active and 1193 placebo doses.

The median frequency for total reactions in each participant was 35\%, with a wide range of $1 \%$ to $95 \%$ in the active group compared with $1 \%$ in the placebo group (range, $0 \%$ to $53 \%$; $P=.02$ ). The most common types of reactions in the active group were local (mostly oral pruritus) and gastrointestinal (mostly abdominal pain), occurring with a median frequency of $16 \%$ and $2 \%$ of active doses, respectively $(P=.006$ and .02$)$. One important note is that of the 458 active doses associated with gastrointestinal symptoms, 2 participants in the active group accounted for about two thirds of the reactions with mild abdominal pain that never required treatment. Reactions involving some combination of gastrointestinal, lower respiratory tract, and skin symptoms were categorized as multiple system reactions. These were rare, occurring with a median frequency of $1 \%$ of active doses versus none in the placebo-treated group $(P=.01)$.

Data on treatment of reactions reveals that diphenhydramine was given with a median frequency of $1 \%$ of active doses compared with $1 \%$ of placebo doses. As with the symptom data, the frequency of diphenhydramine use varied widely in the active group, from never to $58 \%$ of OIT doses. In the active group one participant who was treated for oral itching accounted for one third of diphenhydramine doses administered. Four doses of epinephrine were used to treat reactions in 4 different participants in the active group; 2 of these occurred with the initial in-hospital build-up doses, and the other 2 were with home doses.

There was one participant in each treatment group who experienced an eczema flare. The placebo group participant's flare was managed with topical care along with an oral antibiotic for a skin infection. The active group participant continued to have significant eczema despite aggressive management, which resulted in her early withdrawal from the protocol while receiving the $225-\mathrm{mg}$ OIT dose.

In general, although reactions were common and all active-treated patients experienced at least 1 adverse event, nearly $90 \%$ of all acute reactions were transient and required no treatment. It is also important to note that reactions occurred sporadically at all dose levels, including during maintenance therapy.

\section{Open label-treated group}

Six of the 7 placebo-treated participants elected to receive open-label active treatment. The milk dose threshold was $40 \mathrm{mg}$ in all 6 before OIT. After OIT, the median was $8140 \mathrm{mg}$ (range, $1340-8140 \mathrm{mg} ; P=.03$; Table III and Fig 2). As with the active-treated blinded group, there was a significant change in SPT threshold $(P=.03)$ and IgG4 levels $(P=.03)$. Comparing the open-label active-treated with the blinded active-treated groups, there was no significant difference in change from baseline in any of the SPT, IgE, or IgG antibody measurements.

\section{DISCUSSION}

Among young children, CMA is the most common food allergy, occurring in as many as $2 \%$ to $3 \% .^{18-21}$ Although the prognosis for CMA resolution had been considered good, with up to $85 \%$ tolerating dairy by the age of 3 years, ${ }^{18,19}$ more recent studies show less optimistic 
outcomes. ${ }^{22-26}$ Given that milk allergy is extremely prevalent, might be exhibiting an increasing tendency to persist, is exceedingly difficult to avoid, and has the potential to result in severe and even fatal reactions, ${ }^{5-8}$ it is highly desirable to have some form of therapy to treat this condition.

In this study milk OIT was effective in increasing the threshold for reactions to milk in all treated children. This study is the first done in a double-blind, placebo-controlled fashion in children. before treatment, nearly all individuals had symptoms after the first 40-mg dose at the baseline food challenge, whereas after therapy, the average cumulative dose causing a reaction was $5140 \mathrm{mg}$. The lowest dose causing a reaction in any participant was $1340 \mathrm{mg}$, which still is likely to be protective against the overwhelming majority of accidental exposures. However, it is also important to note that after completion of active treatment, despite an increased threshold, 14 of 18 still exhibited a reaction at the post-OIT food challenge. And even in those who did not react to the post-OIT challenge, it is not clear whether they are fully tolerant or rather transiently desensitized and thus still at risk of future reactions.

Establishing whether patients are desensitized or tolerized through OIT is extremely important. Desensitization should be defined as the ability to tolerate more of an allergenic substance after treatment. The state of desensitization likely requires ongoing exposure to be maintained, and it is completely unclear how long it would take before lack of continued exposure would result in loss of desensitization. Tolerance should be defined as a permanent loss of reactivity to a previously allergenic substance. This can only be proved by completely removing the relevant allergen from an individual's diet for a period of time.

Buchanan et $\mathrm{al}^{9}$ treated 7 patients with $300 \mathrm{mg}$ of egg protein for 24 months. After treatment, 4 of 7 passed an egg challenge. These 4 then restricted egg from their diet for 3 to 4 months and were then rechallenged. Two of the 4 again passed the challenges, but the other 2 were again reactive and at fairly low amounts of egg. Whether this represents inadequate therapy or a subset of patients who will not be capable of tolerance induction remains to be determined. Our current study does not address this issue because all successfully desensitized participants are continuing with daily consumption of dairy as instructed.

With regard to other outcomes, although there were many actively treated participants with large changes in the end-point titration SPT threshold, the median change was not statistically different than that with placebo. It did appear that larger fold changes were associated with higher milk dose thresholds, but this could not be confirmed statistically in this small group. Milk-specific IgE antibody levels remained unchanged, on average, after 3 or 6 months of treatment. In previous studies, significant decreases in food-specific $\operatorname{IgE}$ levels have been reported at time points ranging from 6 to 24 months of therapy. ${ }^{9,11,14,17}$ It is possible that the duration of treatment in our study was not long enough to see a decrease in serum IgE antibody levels. It appeared that higher baseline milk-specific IgE levels were associated with lower post-OIT milk dose thresholds, as well as with greater difficulty in tolerating milk OIT doses, but like the SPT results, this could not be confirmed statistically in this small group.

Milk-specific IgG4 antibody levels exhibited a large increase by the 3-month time point in the actively treated group. Possibly because of sample size, there was no apparent association between the degree of increase in milk-specific IgG4 level and the observed clinical outcome. However, there was large individual variability such that large increases in milk-specific IgG4 antibody levels did not guarantee significantly higher milk thresholds and smaller increases did not exclude higher thresholds. 
There are a number of recent reports in the literature of food-specific OIT, including that to milk, egg, peanut, hazelnut, and fish. ${ }^{10-17}$ However, these reports have varied widely using different modes of delivery (sublingual versus oral/swallowed), widely differing dosing regimens, and variable criteria to define outcomes. In a recent study of OIT by Longo et al, ${ }^{11}$ children with severe milk allergy were gradually escalated to a goal of 5 ounces of milk. After 1 year, one third could tolerate this amount or more, one half tolerated between 1 teaspoon and 5 ounces, and $10 \%$ discontinued treatment because of side effects.

In contrast, Enrique et $\mathrm{l}^{10}$ reported on the use of hazelnut sublingual immunotherapy. These patients were escalated to a maintenance dose in 4 days and treated for 5 months. There was a highly significant increase in hazelnut tolerance in the actively treated group. Five of 11 in the active group compared with 1 of 11 in the placebo group were able to tolerate the entire $20 \mathrm{~g}$ of hazelnut at the posttreatment challenge. However, approximately half of the activetreated participants had a history described as only oral allergy syndrome. Minimal doserelated systemic side effects were reported, and there were no dropouts. The studies by Longo et $\mathrm{al}^{11}$ and Enrique et $\mathrm{al}^{10}$ are representative of the variability in study populations and methodologies used in oral food-specific immunotherapy protocols.

In our study adverse reactions were common, but the risk of a severe reaction fell within the range that we would consider acceptable. Despite the relatively high frequency of reactions of any type ( $45 \%$ of active doses), nearly $90 \%$ were transient reactions that required no treatment. Approximately 1 in 100 active doses resulted in a multisystem reaction, and 1 in 600 resulted in a reaction requiring treatment with epinephrine. Although the ideal for any therapy is no serious reactions, when dealing with highly allergic children, some level of reaction is almost inevitable. It is essential that families considering OIT trials be made aware of these risks, that emergency medications are always available, and that physician coverage be available at all times. Families contemplating such therapy will need to consider whether the benefit of increased, but not necessarily complete, tolerance is worth these risks compared with the alternative of strict avoidance. The majority of prior studies involving orally administered immunotherapy reported less frequent reactions either of a mild or more serious nature. ${ }^{10,12-17}$ Only Longo et al ${ }^{11}$ reported the need for intramuscular epinephrine, and this was only with in-hospital rapid build-up. This contrasts with our experience, in which local symptoms were common in most individuals. Although there was a tendency for symptoms to be more common during build-up, they were still observed during maintenance, even including 1 reaction requiring epinephrine. Possible explanations for these discrepancies could be that our study population was on average more severely allergic, that our subjects were more diligent in reporting symptoms, that we did not require maintenance oral antihistamines as has been done in some other studies, ${ }^{11,12}$ and/or that our dosing protocol was not optimal.

Another issue regarding safety is what appear to be factors associated with a lower threshold for reaction to OIT. This concept was previously raised by Staden et al, ${ }^{17}$ who referred to "'augmentation factors." Although we did not formally study this, it is our impression from this study that both exercise and infectious illnesses can potentially contribute to a reaction at a previously well-tolerated OIT dose.

Future studies should be aimed at improving both the safety and efficacy of this form of treatment. It is possible that smaller incremental increases in doses might lessen reaction frequency or severity. However, because there was no clear preponderance of reactions at or shortly after dose increases, this might not be the case. It is also possible that treatment through the sublingual route, which might allow for much lower doses, would be better tolerated. Having all participants take a daily long-acting antihistamine might help to reduce mild symptoms. 
In conclusion, this study provides evidence that orally administered immunotherapy can result in a significant degree of desensitization to a given food in most allergic patients. Although allergic reactions to OIT doses were common, the majority did not require treatment, and reactions that did require treatment responded well to medications administered by parents. Future studies should aim to establish optimal doses, length of therapy, and route of therapy (oral/swallowed vs sublingual) required to obtain maximal desensitization. Once this is established, further investigation of whether this treatment can induce long-term tolerance or just temporary desensitization will be essential. Finally, given the current uncertainty regarding both safety and efficacy, it is essential that these clinical trials be completed before anyone considers the use of OIT in clinical practice.

\section{Acknowledgments}

Supported by National Institutes of Health (NIH) training grant no. 5T32 AI07007, the Eudowood Foundation, NIH training grant no. 5T32AI007062-30, and Duke Clinical Research Unit/Clinical and Translational Science Award grant no. 5UL1-RR024128-02.

A.W. Burks has consulting arrangements with ActoGeniX, NV, Novartis, McNeil Nutritionals, and Mead Johnson; owns stock in Allertein and MastCell, Inc; is on the advisory board for Dannon Co, Probiotics; is on the speakers' bureau for EpiPen/Dey; is on the data-monitoring committee for Genentech; is on an expert panel for Nutricia; has received research support from the National Institutes of Health (NIH), the Food Allergy and Anaphylaxis Network (FAAN), Gerber, and Mead Johnson; has served as an expert witness in food allergy litigation; and has served as a member of the FAAN, the American College of Allergy, Asthma \& Immunology, the NIH, Hypersensitivity, Autoimmune, and Immunemediated Diseases, and the Journal of Allergy and Clinical Immunology. R. A. Wood has received research support from Genentech and is on the advisory board for the FAAN and Dey.

\section{References}

1. Sampson HA. Food allergy. Part 2: diagnosis and management. J Allergy Clin Immunol. 1999; 103:981-9. [PubMed: 10359874]

2. Sicherer SH, Furlong TJ, Munoz-Furlong A, Burks AW, Sampson HA. A voluntary registry for peanut and tree nut allergy: characteristics of the first 5149 registrants. J Allergy Clin Immunol. 2001; 108:128-32. [PubMed: 11447394]

3. Vander Leek TK, Liu AH, Stefanski K, Blacker B, Bock SA. The natural history of peanut allergy in young children and its association with serum peanut-specific IgE. J Pediatr. 2000; 137:749-55. [PubMed: 11113829]

4. Yu JW, Kagan R, Verreault N, Nicolas N, Joseph L, St Pierre Y, et al. Accidental ingestions in children with peanut allergy. J Allergy Clin Immunol. 2006; 118:466-72. [PubMed: 16890773]

5. Bock SA, Munoz-Furlong A, Sampson HA. Further fatalities caused by anaphylactic reactions to food, 2001-2006. J Allergy Clin Immunol. 2007; 119:1016-8. [PubMed: 17306354]

6. Bock SA, Munoz-Furlong A, Sampson HA. Fatalities due to anaphylactic reactions to foods. J Allergy Clin Immunol. 2001; 107:191-3. [PubMed: 11150011]

7. Pumphrey RS, Gowland MH. Further fatal allergic reactions to food in the United Kingdom, 1999-2006. J Allergy Clin Immunol. 2007; 119:1018-9. [PubMed: 17349682]

8. Sampson HA, Mendelson L, Rosen JP. Fatal and near-fatal anaphylactic reactions to food in children and adolescents. N Engl J Med. 1992; 327:380-4. [PubMed: 1294076]

9. Buchanan AD, Green TD, Jones SM, Scurlock AM, Christie L, Althage KA, et al. Egg oral immunotherapy in nonanaphylactic children with egg allergy. J Allergy Clin Immunol. 2007; 119:199-205. [PubMed: 17208602]

10. Enrique E, Pineda F, Malek T, Bartra J, Basagana M, Tella R, et al. Sublingual immunotherapy for hazelnut food allergy: a randomized, double-blind, placebo-controlled study with a standardized hazelnut extract. J Allergy Clin Immunol. 2005; 116:1073-9. [PubMed: 16275379]

11. Longo G, Barbi E, Berti I, Meneghetti R, Pittalis A, Ronfani L, et al. Specific oral tolerance induction in children with very severe cow's milk-induced reactions. J Allergy Clin Immunol. 2008; 121:343-7. [PubMed: 18158176] 
12. Meglio P, Bartone E, Plantamura M, Arabito E, Giampietro PG. A protocol for oral desensitization in children with IgE-mediated cow's milk allergy. Allergy. 2004; 59:980-7. [PubMed: 15291907]

13. Morisset M, Moneret-Vautrin DA, Guenard L, Cuny JM, Frentz P, Hatahet R, et al. Oral desensitization in children with milk and egg allergies obtains recovery in a significant proportion of cases. A randomized study in 60 children with cow's milk allergy and 90 children with egg allergy. Eur Ann Allergy Clin Immunol. 2007; 39:12-9. [PubMed: 17375736]

14. Patriarca G, Nucera E, Pollastrini E, Roncallo C, De Pasquale T, Lombardo C, et al. Oral specific desensitization in food-allergic children. Dig Dis Sci. 2007; 52:1662-72. [PubMed: 17245630]

15. Patriarca G, Nucera E, Roncallo C, Pollastrini E, Bartolozzi F, De Pasquale T, et al. Oral desensitizing treatment in food allergy: clinical and immunological results. Aliment Pharmacol Ther. 2003; 17:459-65. [PubMed: 12562461]

16. Patriarca G, Schiavino D, Nucera E, Schinco G, Milani A, Gasbarrini GB. Food allergy in children: Results of a standardized protocol for oral desensitization. Hepatogastroenterology. 1998; 45:52-8. [PubMed: 9496487]

17. Staden U, Rolinck-Werninghaus C, Brewe F, Wahn U, Niggemann B, Beyer K. Specific oral tolerance induction in food allergy in children: Efficacy and clinical patterns of reaction. Allergy. 2007; 62:1261-9. [PubMed: 17919140]

18. Bock SA. Prospective appraisal of complaints of adverse reactions to foods in children during the first 3 years of life. Pediatrics. 1987; 79:683-8. [PubMed: 3575022]

19. Host A, Halken S. A prospective study of cow milk allergy in Danish infants during the first 3 years of life. clinical course in relation to clinical and immunological type of hypersensitivity reaction. Allergy. 1990; 45:587-96. [PubMed: 2288394]

20. Saarinen KM, Juntunen-Backman K, Jarvenpaa AL, Kuitunen P, Lope L, Renlund M, et al. Supplementary feeding in maternity hospitals and the risk of cow's milk allergy: a prospective study of 6209 infants. J Allergy Clin Immunol. 1999; 104:457-61. [PubMed: 10452771]

21. Schrander JJ, van den Bogart JP, Forget PP, Schrander-Stumpel CT, Kuijten RH, Kester AD. Cow's milk protein intolerance in infants under 1 year of age: a prospective epidemiological study. Eur J Pediatr. 1993; 152:640-4. [PubMed: 8404966]

22. Bishop JM, Hill DJ, Hosking CS. Natural history of cow milk allergy: clinical outcome. J Pediatr. 1990; 116:862-7. [PubMed: 2348289]

23. Hill DJ, Davidson GP, Cameron DJ, Barnes GL. The spectrum of cow's milk allergy in childhood. clinical, gastroenterological and immunological studies. Acta Paediatr Scand. 1979; 68:847-52. [PubMed: 539407]

24. Hill DJ, Firer MA, Ball G, Hosking CS. Natural history of cows' milk allergy in children: immunological outcome over 2 years. Clin Exp Allergy. 1993; 23:124-31. [PubMed: 8448679]

25. Saarinen KM, Pelkonen AS, Makela MJ, Savilahti E. Clinical course and prognosis of cow's milk allergy are dependent on milk-specific IgE status. J Allergy Clin Immunol. 2005; 116:869-75. [PubMed: 16210063]

26. Skripak JM, Matsui EC, Mudd K, Wood RA. The natural history of IgE-mediated cow's milk allergy. J Allergy Clin Immunol. 2007; 120:1172-7. [PubMed: 17935766]

\section{Abbreviations used}

$\begin{array}{ll}\text { CM } & \text { Cow's milk } \\ \text { CMA } & \text { Cow's milk allergy } \\ \text { DBPCFC } & \text { Double-blind, placebo-controlled food challenge } \\ \text { OIT } & \text { Oral immunotherapy } \\ \text { SPT } & \text { Skin prick test }\end{array}$




\section{Clinical implications}

Milk OIT is effective in desensitizing allergic children. Further studies are needed to clarify optimal dosing and duration of therapy, determine whether permanent tolerance is achievable, and reduce adverse reactions. 


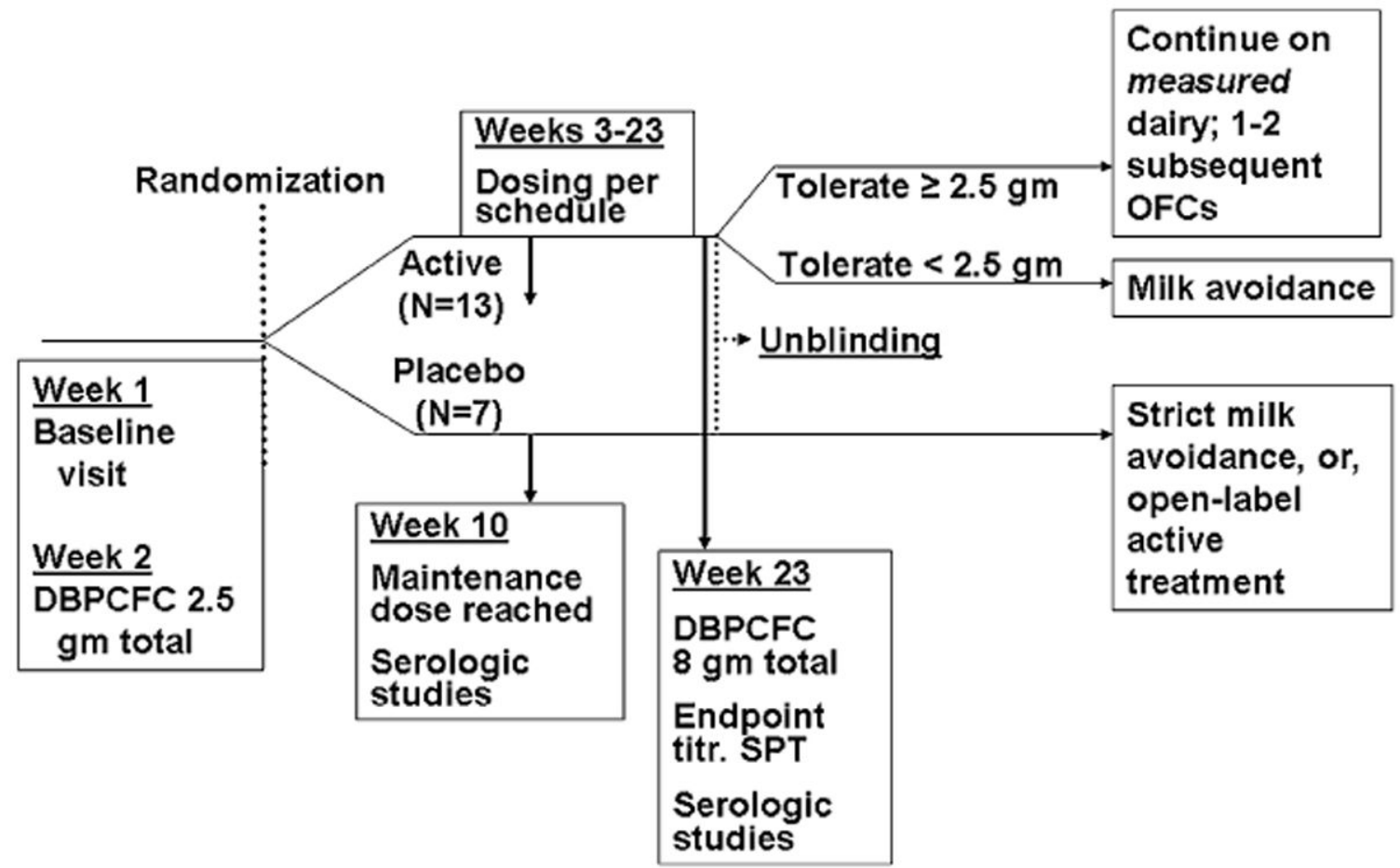

FIG 1.

Study timeline. Key features and overall timeframe are shown. $O F C$, Oral food challenge. 

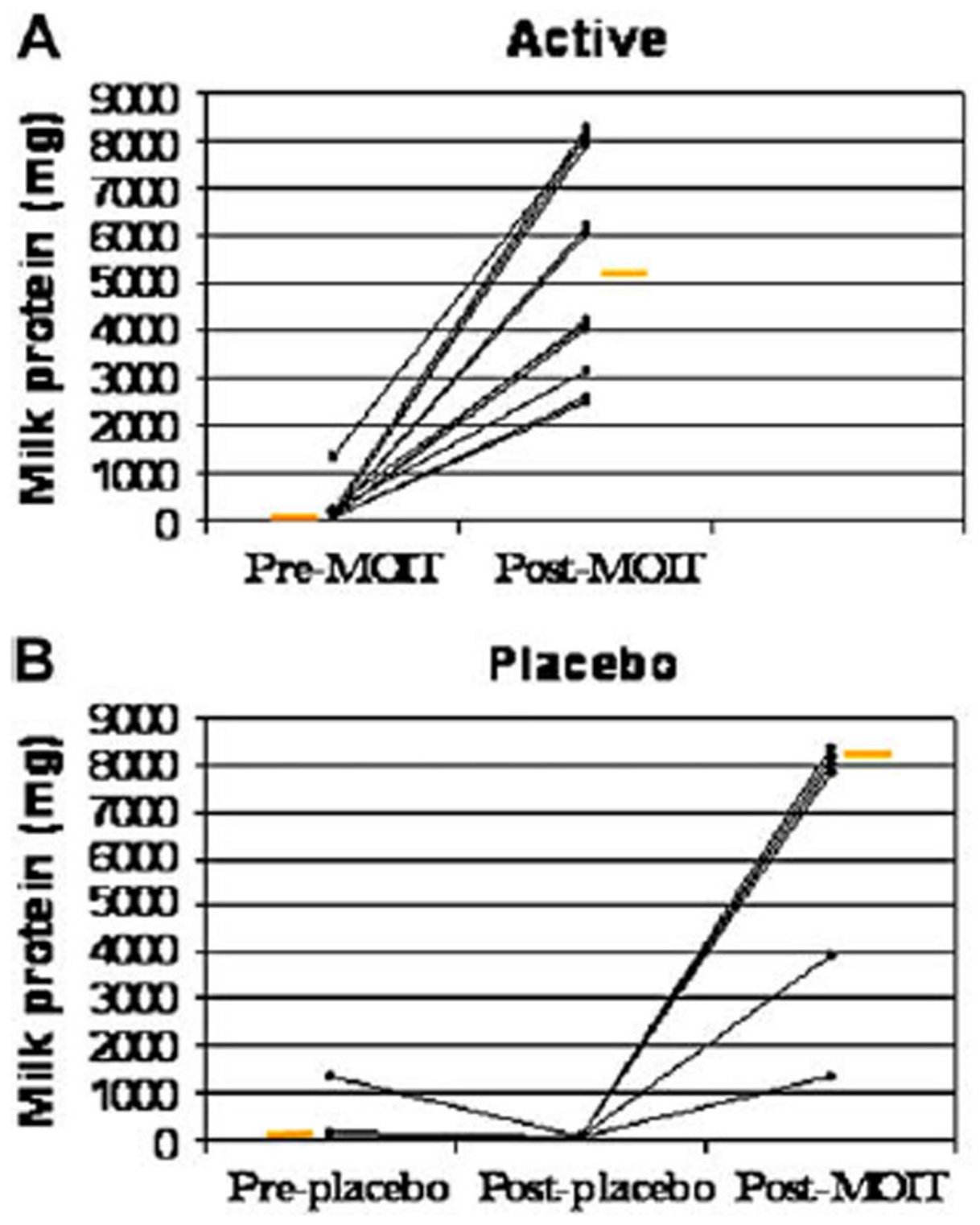

FIG 2.

Change in milk dose threshold. A, Threshold change in active group $(P=.002)$. B, Threshold change in placebo group $(P=.16)$. Change in threshold after open-label active treatment in the placebo group is also shown in Fig 2, $B$ (from after placebo to after milk OIT). Change in threshold for active versus placebo groups, $P=.0003$. Orange bars represent medians. MOIT, Milk OIT. 


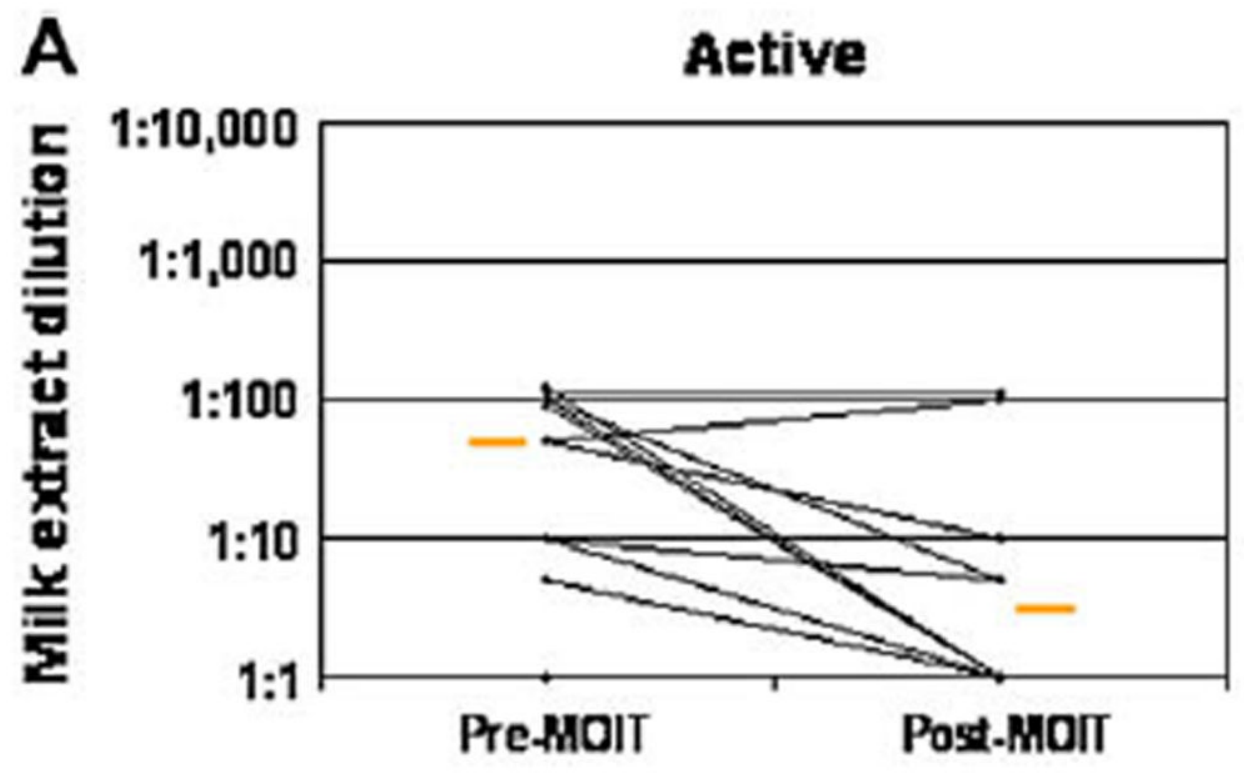

Placebo

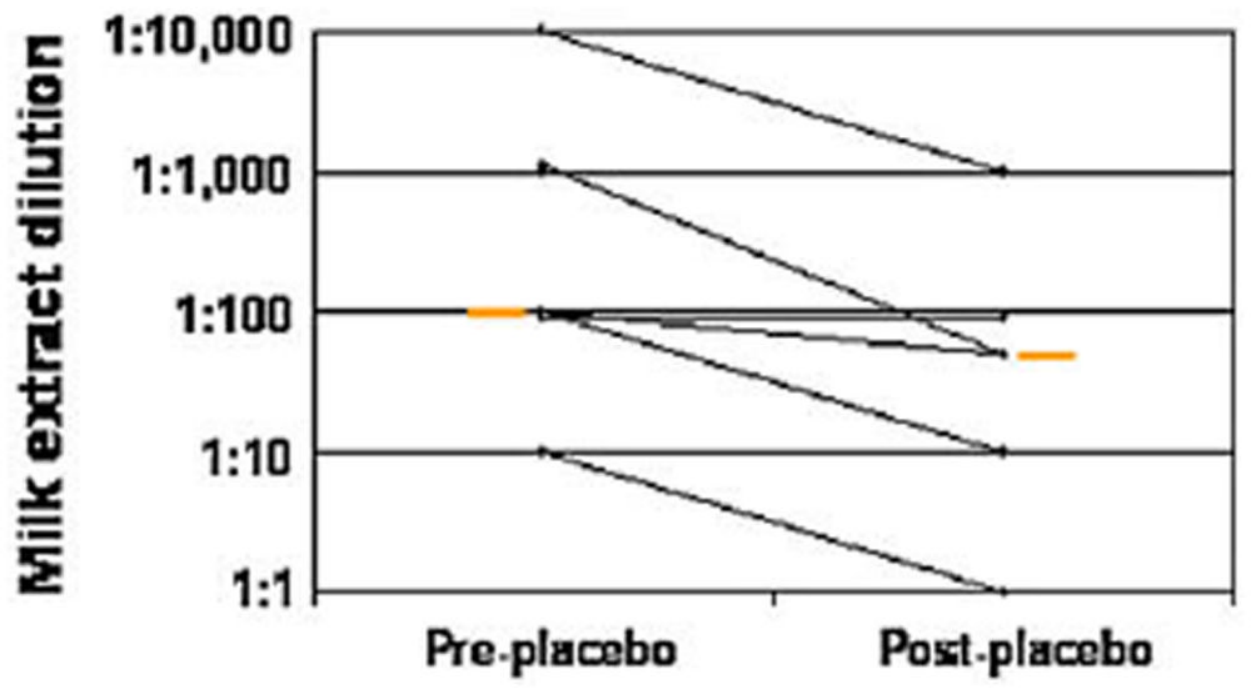

FIG 3.

Change in end-point titration skin prick testing. A, Threshold change in active group $(P=$. 03). B, Threshold change in placebo group $(P=.03)$. Change in threshold for active versus placebo groups, $P=.54$. Orange bars represent medians. MOIT, Milk OIT. 

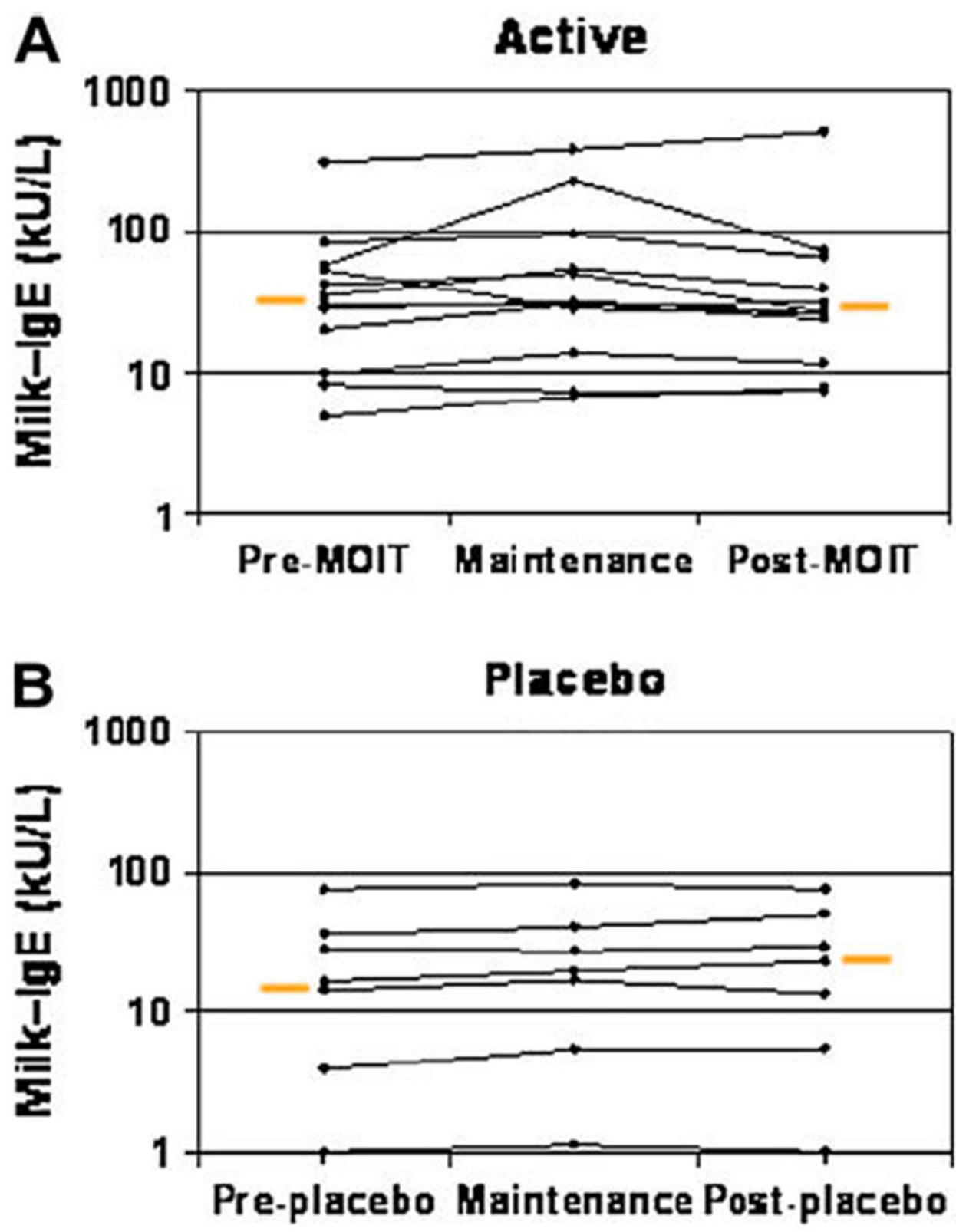

FIG 4.

Change in milk-specific IgE levels. Results are shown before milk OIT (MOIT), at maintenance, and after MOIT for the active group $(P=.75 ; \mathbf{A})$ and placebo group $(P=.46$; B). Change in threshold for active versus placebo groups, $P=.87$. Orange bars represent medians. 

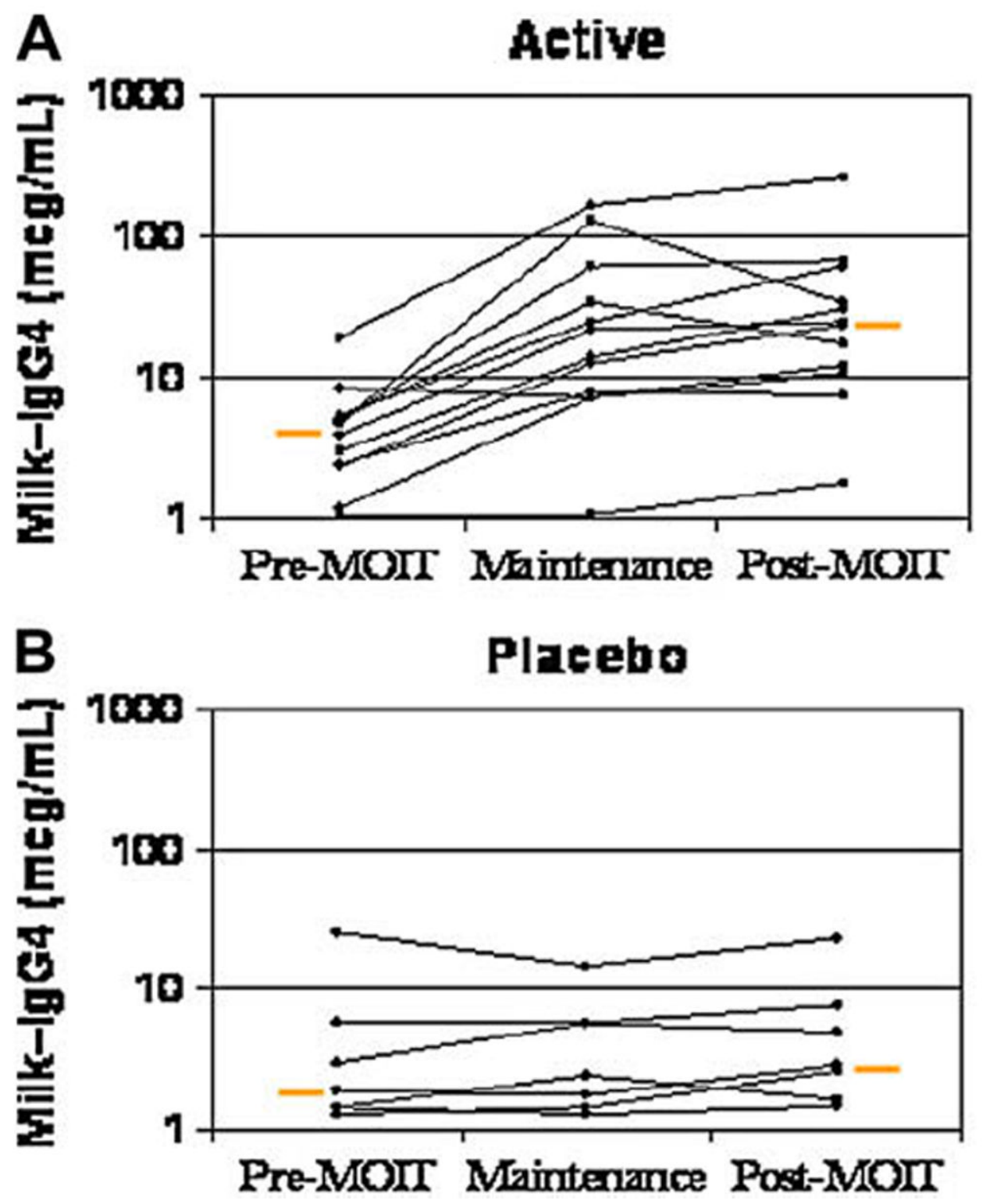

FIG 5.

Change in milk-specific IgG4 levels. Results are shown before milk OIT (MOIT), at maintenance, and after MOIT for the active group $(P=.002 ; \mathbf{A})$ and placebo group $(P=.6$; B). Change in threshold for active versus placebo groups, $P=.001$. Orange bars represent medians. 


\section{TABLE I}

OIT dosing in milligrams of CM protein

\begin{tabular}{lc}
\hline Initial build-up day & Every $\mathbf{1}$ to $\mathbf{2}$ week dose increases \\
\hline 0.4 & 75 \\
\hline 0.8 & 100 \\
\hline 1.5 & 130 \\
\hline 3 & 170 \\
\hline 6 & 225 \\
\hline $12^{*}$ & 295 \\
\hline 25 & 385 \\
\hline 50 & 500 \\
\hline$*$ & \\
\hline
\end{tabular}


TABLE II

Demographics of study participants randomized to active or placebo treatment

\begin{tabular}{lccc}
\hline Characteristics & Active-treated group $(\mathbf{n}=\mathbf{1 3})$ & Placebo-treated group $(\mathbf{n}=\mathbf{7})$ & $\boldsymbol{P}$ value \\
\hline Male sex, no. $(\%)$ & $8(62)$ & $4(57)$ & 1.0 \\
\hline Age (y), mean (SD) & $9.3(3.3)$ & $10.2(3.3)$ & .5 \\
\hline Hx/o eczema, no. $(\%)$ & $7(54)$ & $4(57)$ & 1.0 \\
\hline Current eczema, no. $(\%)$ & $4(31)$ & $2(29)$ & 1.0 \\
\hline Hx/o asthma, no. $(\%)$ & $12(92)$ & $5(71)$ & .27 \\
\hline Current asthma, no. $(\%)$ & $9(69)$ & $3(43)$ & .36 \\
\hline Hx/o other FA, no. $(\%)$ & $10(77)$ & $5(71)$ & 1.0 \\
\hline No. of other current FA, median (range) & $2(0-8)$ & $2(0-5)$ & .8 \\
\hline Baseline CM IgE (kUA/L), median (range) & $34.8(4.86-314)$ & $14.6(0.93-133.4)$ & .19 \\
\hline
\end{tabular}

$H x / o$, History of; $F A$, food allergy. 


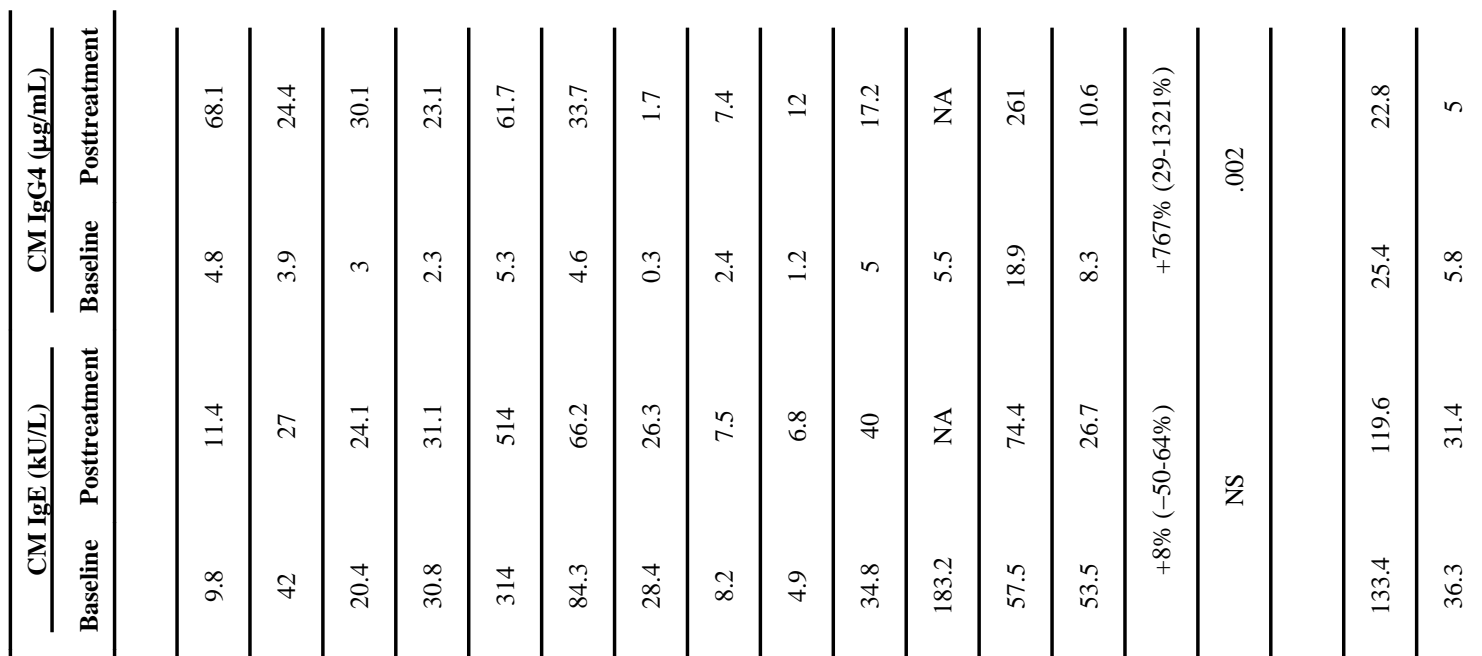

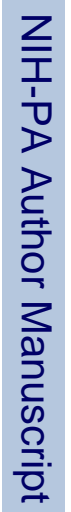

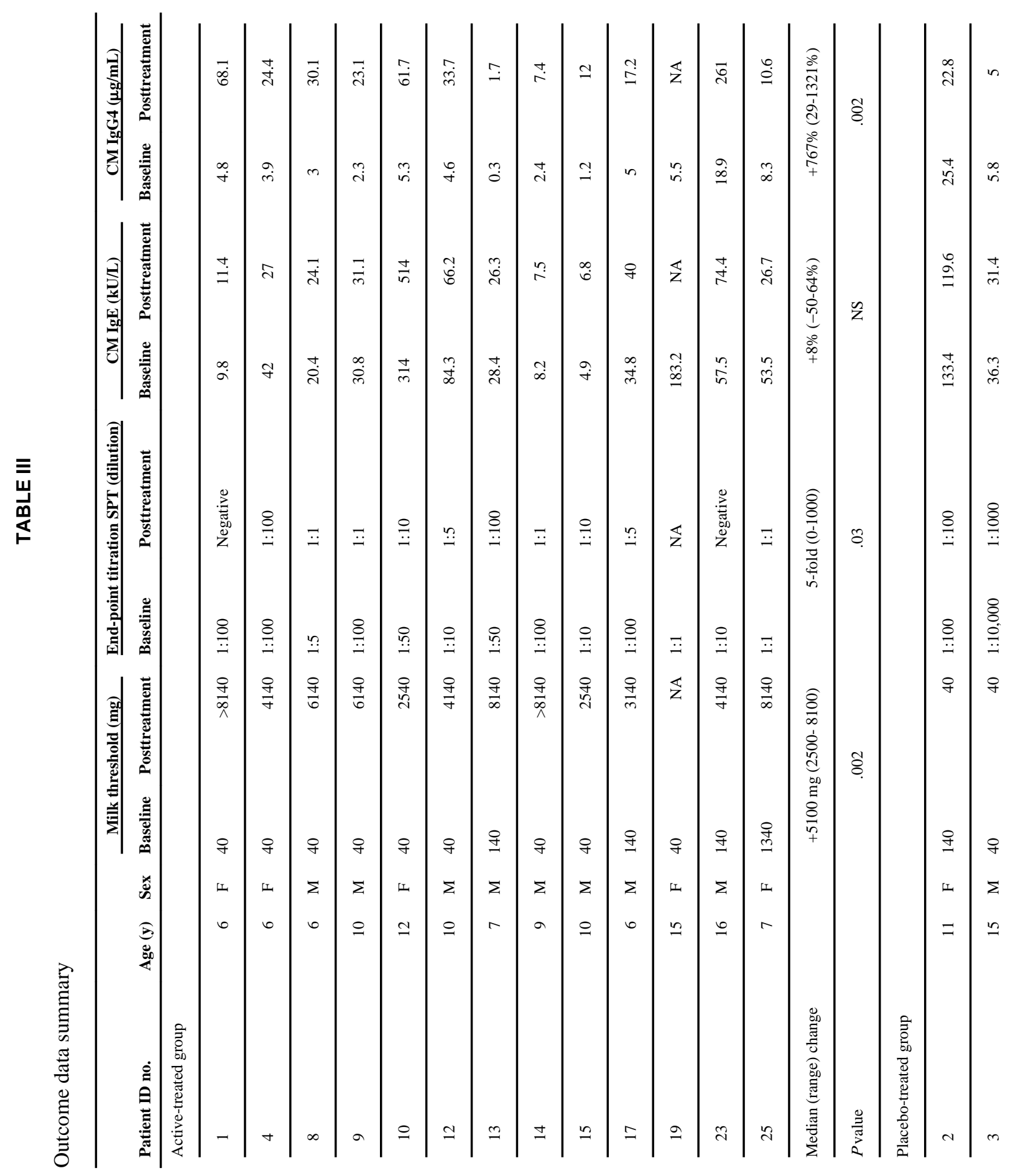




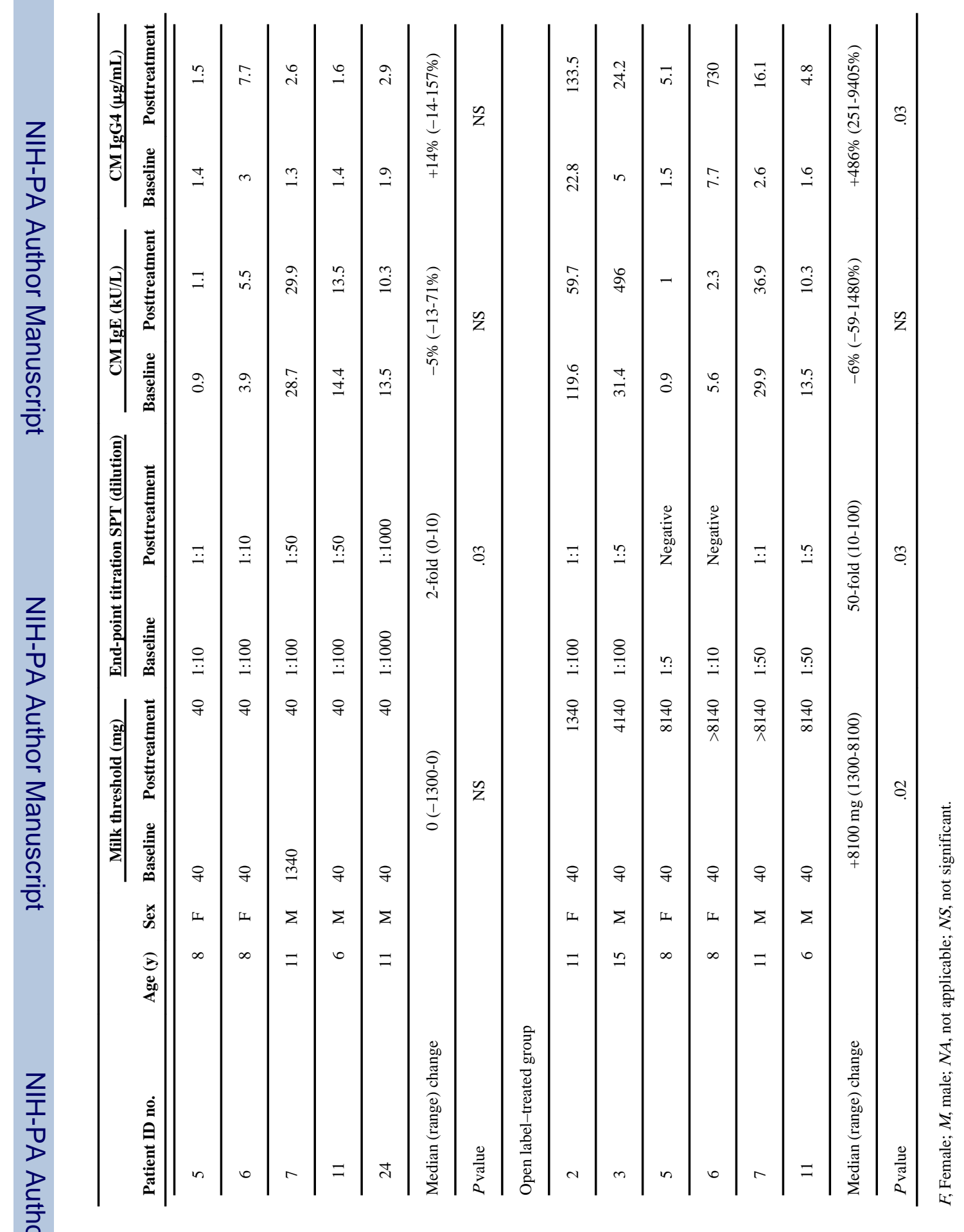




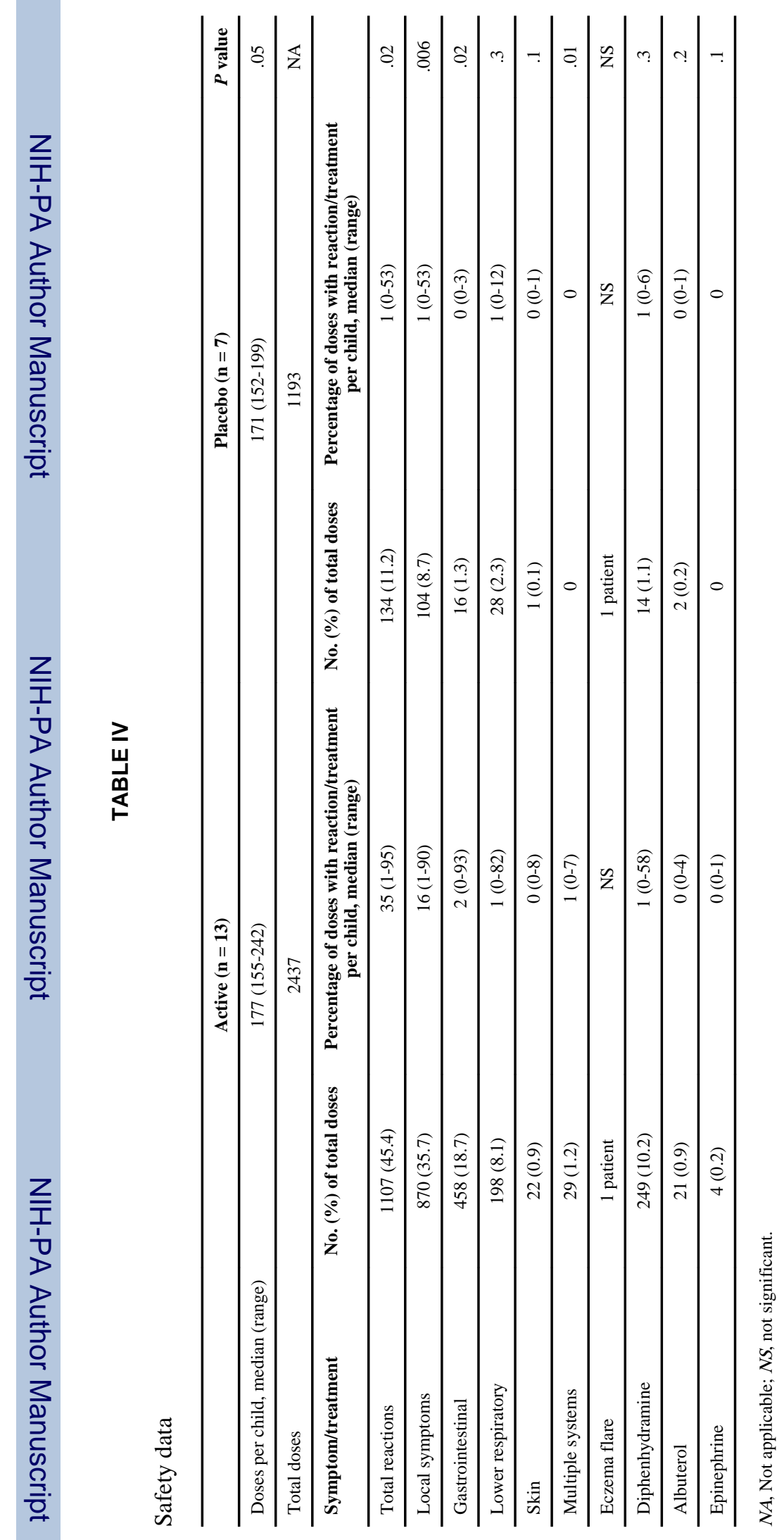

